\title{
VR Rehearse \& Perform - A platform for rehearsing in Virtual Reality
}

\author{
Vali Lalioti* \\ v.lalioti@arts.ac.uk \\ University of the Arts London \\ Creative Computing Institute \\ London, United Kingdom
}

\section{Ragnar Hrafnkelsson \\ ragnar@reactifymusic.com \\ Reactify Music \\ London, United Kingdom}

\author{
Sophia Ppali* \\ sp815@kent.ac.uk
}

University of the Arts London

Creative Computing Institute

London, United Kingdom

University of Kent

Engineering and Digital Arts

Canterbury, United Kingdom

\section{Mick Grierson}

m.grierson@arts.ac.uk

University of the Arts London

Creative Computing Institute

London, United Kingdom

\author{
Andrew Thomas \\ andrew.thomas@rcm.ac.uk \\ Royal College of Music \\ London, United Kingdom
}

\author{
B. S. Wohl \\ b.wohl@arts.ac.uk \\ University of the Arts \\ Creative Computing Institute \\ London, United Kingdom
}

\author{
Alexandra Covaci \\ a.covaci@kent.ac.uk \\ University of Kent \\ Engineering and Digital Arts \\ Canterbury, United Kingdom
}

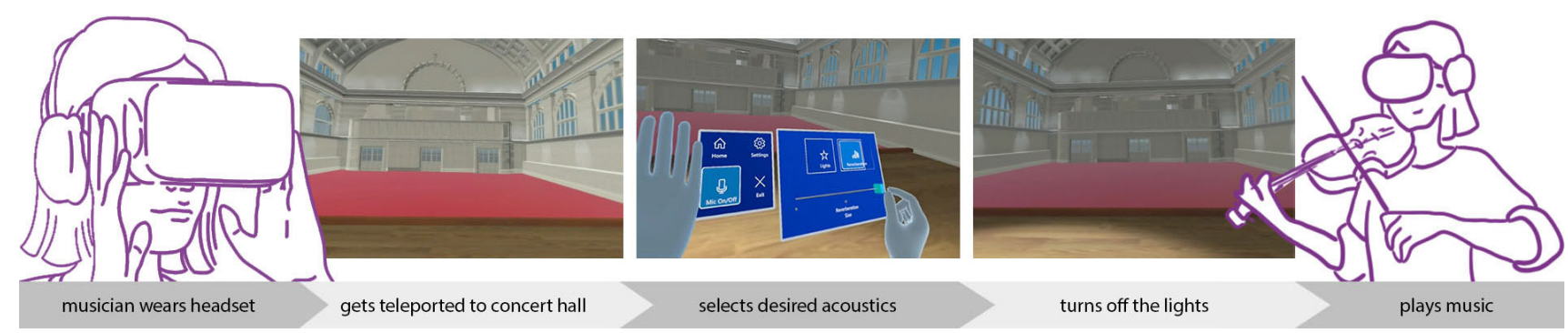

Figure 1: VR Rehearse \& Perform - a Virtual Reality system to support musicians' practice.

\begin{abstract}
In this paper, we propose VR Rehearse \& Perform - a Virtual Reality application for enhancing the rehearsal efforts of performers by providing them access to accurate recreations - both visual and acoustical - of iconic concert venues.
\end{abstract}

\section{KEYWORDS}

Virtual Reality, Rehearsing, Musicians, Acoustic Environments

\footnotetext{
*Both authors contributed equally to this research.

Permission to make digital or hard copies of part or all of this work for personal or classroom use is granted without fee provided that copies are not made or distributed for profit or commercial advantage and that copies bear this notice and the full citation on the first page. Copyrights for third-party components of this work must be honored.

For all other uses, contact the owner/author(s).

VRST '21, December 8-10, 2021, Osaka, Japan

(C) 2021 Copyright held by the owner/author(s)

ACM ISBN 978-1-4503-9092-7/21/12.

https://doi.org/10.1145/3489849.3489896
}

ACM Reference Format:

Vali Lalioti, Sophia Ppali, Andrew Thomas, Ragnar Hrafnkelsson, Mick Grierson, Chee Siang Ang, B. S. Wohl, and Alexandra Covaci. 2021. VR Rehearse \& Perform - A platform for rehearsing in Virtual Reality. In 27th ACM Symposium on Virtual Reality Software and Technology (VRST '21), December 8-10, 2021, Osaka, Japan. ACM, New York, NY, USA, 3 pages. https://doi.org/10.1145/3489849.3489896

\section{INTRODUCTION}

The COVID-19 pandemic has heavily impacted artists' ability to rehearse and perform [7]. Musicians and performers are training from home spaces which bear no resemblance - visual nor acoustical - to rehearsing and concert venues. Musical rehearsal rooms act as an acoustical transformer and have a significant influence on improving the musicians' ability to perceive and present their unique sound [4]. Therefore, practicing from home can affect performers' unconscious adjustment to source levels [1, 2], severely limiting rehearsing effectiveness. The current context has only exacerbated 
an existing problem, as access to venues, particularly iconic ones, is limited in terms of time, and often comes at a high cost.

Because of its ability to bring users in new fictional worlds, Virtual Reality (VR) is used in a variety of applications, across numerous domains of knowledge [6]. However, VR applications have not addressed the enhancement of musicians' practice. In this paper, we address this gap and we propose VR Rehearse \& Perform a proof of concept of a VR solution aimed to support performers' rehearsal efforts by exposing them to accurate simulation of the visual and acoustic features of concert venues. Specifically, with $V R$ Rehearse \& Perform, we aim to deliver accessible concert hall-like immersive experiences where musicians have the illusion of being on stage and benefit from realistic soundscapes based on real-time 3D sound and reverberation.

\section{VR REHEARSE \& PERFORM}

In VR Rehearse \& Perform we followed a research through design approach [3], and together with experts in performance and musicians, we explored the potential for technology inclusion in music practice. The following design requirements were gathered: 1) create an audio-visual simulation which combines auralisations with visualizations of an iconic concert hall; 2) allow the customisation of both visual and acoustic stage parameters; 3) use hand tracking to interact with the VR application, thus allowing musicians to play their instruments freely.

Based on the design requirements we developed the VR Rehearse \& Perform prototype (Figure 1), for the Oculus Quest, using Unity 2020.3.7f1 and C\# as the main programming language. In VR Research \& Perform, musicians are positioned in the middle of the stage of a 3D reconstructed, concert hall. They can interact with the lights of the auditorium - adjusting their intensity - and choose between four types of acoustics (three with different degrees of Plausibility and one Authentic - the Room Impulse Response (RIR) of the hall). The scene has a real-time microphone input feature which enables the performer to sing or play an instrument and listen back to their performance rendered in real-time based on the type of acoustics they select. Hand tracking was used as the main form of user interaction in our prototype, using Microsoft's Mixed Reality Toolkit.

\section{PRELIMINARY USER EVALUATION}

\subsection{Participants and procedure.}

We invited eight musicians (5 male and 3 female, age: 21-40, 4 vocalists, 1 guitarist, 1 keyboard player, 2 piano players) to evaluate the potential of VR Rehearse \& Perform to support their music practice. All the participants signed an informed consent, and gave us permission to use the screen/audio recordings from their sessions. We asked participants to play their instruments while interacting with VR Rehearse \& Perform (see Figure 2), followed by a short interview.

\subsection{Preliminary results.}

Overall, participants had the feeling of "being there" in the concert hall and saw potential benefits of playing in VR. The user evaluations showed that VR Rehearse \& Perform has potential to enhance musicians' rehearsals by allowing them to adjust to the acoustics and reverberation of performance halls. The application is also

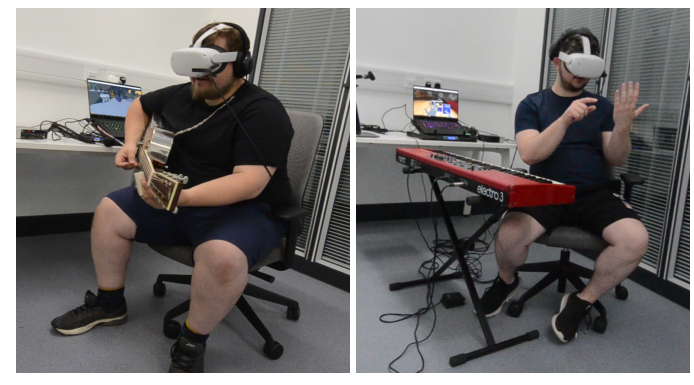

Figure 2: Performer playing music in VR Rehearse \& Perform (left) and using hand-tracking to change the settings (right)

well suited for musicians who want to improvise and experiment within a new venue, hence potentially developing performances explicitly designed for specific venues' acoustics. However, one of the limitations of our system is that participants could not see their instrument while playing in VR. This was especially problematic for two of the pianists in our study, therefore further work is needed to incorporate instruments in the software.

We observed that real-time reverberation enhanced the usefulness of VR as a performance preparation tool and was perceived as important for immersion, increasing the value of the application. The introduction of the Authentic acoustics came with significant latency due to the limited processing power of the Oculus Quest. The Plausible acoustics were better received, with musicians finding the sound reproduction realistic and enjoying the options that emulate the general reverberation characteristics of a full (shorter decay) or empty (longer decay) concert hall. This suggests that for musicians, approximations of real environment acoustics in VR might work better than accurate reproductions, but further investigation is required to identify the threshold for the evaluation of acoustics. Moreover, it is important to explore the factors (e.g., visual cues, time of exposure to the VR environment, genre of music, experience of the musicians, etc.) that could help musicians adapt to the inherent latency produced by such systems [5].

\section{ACKNOWLEDGMENTS}

The project reported in this article was supported by the United Kingdom's Research and Innovation department, funded by Innovate UK, Grant no: 58128. The research was also supported by HEartS Professional, a project funded by the United Kingdom's Arts and Humanities Research Council (AHRC), Grant no: AH/V013874/1.

\section{REFERENCES}

[1] Judith Brereton, Damian Murphy, and David M Howard. 2011. Evaluating the Auralization of Performance Spaces and its Effect on Singing Performance. In Audio Engineering Society Convention 130. Audio Engineering Society.

[2] Anders C Gade. 1989. Investigations of musicians' room acoustic conditions in concert halls. Part I: methods and laboratory experiments. Acta Acustica united with Acustica 69, 5 (1989), 193-203.

[3] William Gaver. 2012. What should we expect from research through design?. In Proceedings of the SIGCHI conference on human factors in computing systems. 937-946.

[4] Doyuen Ko and Wieslaw Woszczyk. 2018. virtual acoustics for musicians: subjective evaluation of a virtual acoustic system in performance of string quartets. Journal of the Audio Engineering Society 66, 9 (2018), 712-723. 
[5] Luca Remaggi, Hansung Kim, Philip JB Jackson, and Adrian Hilton. 2019. Reproducing real world acoustics in virtual reality using spherical cameras. In Audio Engineering Society Conference: 2019 AES International Conference on Immersive and Interactive Audio. Audio Engineering Society.

[6] Mel Slater and Maria V Sanchez-Vives. 2016. Enhancing our lives with immersive virtual reality. Frontiers in Robotics and AI 3 (2016), 74.
[7] Neta Spiro, Rosie Perkins, Sasha Kaye, Urszula Tymoszuk, Adele Mason-Bertrand, Isabelle Cossette, Solange Glasser, and Aaron Williamon. 2021. The Effects of COVID-19 Lockdown 1.0 on Working Patterns, Income, and Wellbeing among 1 Performing Arts Professionals in the United Kingdom (April-June 2020). Frontiers in Psychology (2021), 1-30. https://doi.org/10.3389/fpsyg.2020.594086 